\title{
Materials Design and Osteoinduction Characteristics of Biomimetic and Functionally Graded Apatites
}

\author{
Toshiyuki Akazawa $^{1}$, Masaru Murata ${ }^{2}$ and Junichi Tazaki ${ }^{2}$
}

${ }^{1}$ Department of Materials Technology, Hokkaido Industrial Research Institute, Japan
${ }^{2} 2$ nd Department of Oral Surgery, School of Dentistry, Health Sciences University of Hokkaido, Japan

\begin{abstract}
Bioabsorbable and functionally graded apatites (fg-HAp) were designed using bovine bone by the calcination and partial dissolution-precipitation methods. The fg-HAp ceramics had the specified surface structure and pore size distribution derived from living body. The pore structure of the ceramics with specific surface areas of 30-40 $\mathrm{m}^{2} ¥ \mathrm{~g}^{-1}$ and porosities of $60-80 \%$ was divided into a macro-pore originating from spongy bone and a micro-pore related to body fluid permeation and blood permeability. Recombinant human bone morphogenetic protein-2 (rhBMP-2) solution was added to fg-HAp ceramics to obtain rhBMP-2-loaded fgHAp (rhBMP-2/fg-HAp) ceramics exhibiting osteoinduction. The fg-HAp and rhBMP-2/fg-HAp ceramics were implanted to the subcutaneous tissue of rats. At 4 weeks after the implantation, for the fg-HAp, body fluid and albumin permeated the bulk region of the ceramics through the micro-pores, and surface- and bulk-degradations of the HAp grains proceeded. At 12 weeks, the fg-HAp ceramics were completely biodegraded and bioabsorbed. For the rhBMP-2/ fg-HAp, at 4 weeks after the implantation, some pieces of fg-HAp were incorporated into the induced bone and fatty marrow, suggesting that osteoinduction occurred in conjunction with bone remodeling. The rhBMP-2/fg-HAp ceramics would be newly biomimetic materials with excellent blood permeability and osteoinduction.
\end{abstract}

Key words: Bioabsorption; Biodegradation; Blood permeability; Functionally graded apatites; Osteoinduction

\begin{abstract}
Introduction
Hydroxyapatite (HAp: $\mathrm{Ca}_{10}\left(\mathrm{PO}_{4}\right)_{6}(\mathrm{OH})_{2}$ ) has been widely used for dental and medical implants because of its excellent biocompatibility and osteoconduction ${ }^{1,2}$. In recent years, coping with necessity for patients, i.e., implanting location or situation, development and application of tailored HAp ceramics which can take a reasonable balance between absorption rate and mechanical strength in a living body have been expected ${ }^{3}$. HAp ceramics synthesized from reagents show very low bioabsorption characteristics in implanted regions even after several years. Therefore, commercial HAp ceramics cannot always be satisfactory materials for bone regeneration and long-time use $e^{4}$. Responding to the background, we focus on the development of biomimetic HAp ceramics having controlled bioabsorption and bone remodeling ${ }^{5,6)}$. HAp ceramics made of natural bone have physical and chemical properties of raw materials such as pore structure and small amounts of metal ions ${ }^{7}$. For instance, bovine bone-originated HAp (designated as b-HAp), which was calcined at $800-1100 \bullet$, can give high porosities and interconnecting pore system as well as appropriate mechanical strength ${ }^{8,9)}$. Surface modification of the calcined HAp ceramics might bring the control of absorption rate of HAp ceramic blocks in a living body.

Bone morphogenetic protein (BMP) is known as one of bone formation factors accelerating osteoinduction, and it has been added to various scafford materials because of its novel ability to induce bone and cartilage in intramuscular and subcutaneous tissues $^{10-12)}$. Oda et al. ${ }^{13)}$ and Takahashi et al. ${ }^{14)}$ demonstrated that interconnected porous HAp and calcium phosphate ceramics
\end{abstract}

Correspondence to Toshiyuki Akazawa, Department of Materials Technology, Hokkaido Industrial Research Institute, Nishi-11, Kita-19, Kita-ku, Sapporo, 060-0819 Japan, Tel: 81-11-747-2932, Fax: 81-11-7264057, e-mail:akazawa@hokkaido-iri.go.jp would be superior carriers of recombinant human BMP-2 (rhBMP2 ) in ectopic and orthotopic sites. Gradations in the grain size and crystallinity of fg-HAp plus rhBMP-2-addition might achieve the desired bio-absorption and osteoinduction characteristics.

The first purpose of this study is to securely design biomimetic and functionally graded HAp (designated as fg-HAp) ceramics using bovine bone by the calcination and partial dissolutionprecipitation methods. The second purpose is to design rhBMP2- loaded fg-HAp (designated as rhBMP-2/fg-HAp) ceramics with an excellent osteoinduction. The third purpose is to histologically and immunohistochemically evaluate the bioabsorption and osteoinduction characteristics of the two ceramics.

\begin{abstract}
Materials and Method
Design of fg-HAp ceramics derived from bovine bone

Spongy and cortical bovine femur bones were used as starting materials, boiled and calcined at $800-1100^{\circ} \mathrm{C}$ for $24 \mathrm{~h}$ in air to obtain crystalline b-HAp ceramics ${ }^{9}$. By the calcination process, all prion proteins and organic residues of bovine bone were completely burned out. The pulverized cortical b-HAp was completely dissolved into a $\mathrm{HNO}_{3}$ solution, while the spongy bHAp was partially dissolved into another $\mathrm{HNO}_{3}$ solution. After the solutions were mixed, $\mathrm{NH}_{3}$ solution was added and reprecipitated HAp (designated as r-HAp) crystals were carefully precipitated on macro-pores and micro-pores of spongy b-HAp at $\mathrm{pH} 10.5$ and $25^{\circ} \mathrm{C}^{5}$. The modified spongy b-HAp was aged for $24 \mathrm{~h}$ to fabricate a structure of fg-HAp. The fg-HAp ceramics were filtrated and washed with distilled water and dried at $120^{\circ} \mathrm{C}$ . To obtain rhBMP-2/fg-HAp ceramics, rhBMP-2(5 $\mu$ g) (supplied by Yamanouchi Pharmaceutical Co Ltd, Tokyo, Japan) solution was added to fg-HAp ceramics blocks (3x3x3mm) in a sterilized dish.
\end{abstract}




\section{J.Hard Tissue Biology.14(2)Proceeding,2005}

\section{Characterization of biomimetic fg-HAp ceramics}

The crystalline phase of the samples was identified by Micro$\mathrm{X}$-ray diffraction (Micro-XRD) using $\mathrm{Cu} \mathrm{K}_{\mathrm{a} 1}$ radiation. Scanning electron microscopic (SEM) photographs of the fg-HAp ceramics were taken to observe the morphology and microstructure. The porosities of the ceramics were determined by the water displacement method. The BET-specific surface areas were measured from $\mathrm{N}_{2}-$ adsorption at $-196^{\circ} \mathrm{C}$. The curves of pore size distribution were measured by the mercury permeation method for macro-pores and the $\mathrm{N}_{2}$-adsorption method for micropores.

Histological and immunochemical observations of fg-HAp and rhBMP-2/fg-HAp explants

The fg-HAp and rhBMP-2/fg-HAp ceramics blocks were implanted into the subcutaneous tissues of the back region in 4week-old male Wistar rats. In all procedures, the international guidelines for experiments on animals were followed ${ }^{4,6)}$. At 4, 8, and 12 weeks after implantation, these samples were explanted. The specimens were fixed in neutral buffered formalin, decalcified with formic acid, embedded in paraffin, sectioned and stained with hematoxylin and eosin (HE). They were histologically evaluated using an optical microscope. To investigate the eosinophilic areas in HE sections of the implanted ceramics blocks, immunohistochemical staining was carried out. The specimens were immunostained with polyclonal rabbit antibody against rat albumin (IgG fraction rabbit anti-rat albumin, Inter-Cell Technologies, Inc., USA) using the avidin-biotin complex method. The sections were treated with 3,3'- diaminobenzidine in Tris buffer solution containing hydrogen peroxide for visualization of reaction products. Incubations with normal rabbit IgG or without the primary antibody were used as negative controls.

\section{Results \\ Characterization of biomimetic $f g-H A p$ ceramics with the specified pore structure}

Appearance of fg-HAp ceramics blocks ( $3 \times 3 \times 3 \mathrm{~mm})$ is shown in Fig.1. The fg-HAp ceramics blocks had sufficient strength for handling and operation. The Micro-XRD analyses indicated that the degree of crystallinity of the single phase HAp was gradually distributed from the surface layer of the macro-pore wall to the bulk region of the b-HAp body structure ${ }^{5}$. Figure 2 shows SEM photographs of the fg-HAp ceramics prepared by the calcination at $800^{\circ} \mathrm{C}$ and partial dissolution-precipitation. Macro-pore sizes of 100-600 $\mu \mathrm{m}$ (Fig.1a) and grain sizes of 0.3-0.5 $\mu \mathrm{m}$ in the surface and the macro-pores (Fig.1b) were observed. When the r-HAp crystals were precipitated on the pore wall surface in the macropores and micro-pores of the b-HAp, the microstructures of the fg-HAp ceramics would change to spherical moss-like grains forming needle-like microcrystals of the r-HAp. The specific surface areas, total pore volumes, and porosities of the ceramics were $30-40 \mathrm{~m}^{2} \square \mathrm{g}^{-1}, 0.379-0.390 \mathrm{~cm}^{3} \square \mathrm{g}^{-1}$, and $60-80 \%$, respectively. Comparing with the curves of the pore size distribution for the b-HAp and fg-HAp ceramics, pore volumes in the micro-pore sizes of 10-160 nm were clearly recognized for only the fg-HAp ceramics.

Bioabsorption and osteoinduction of fg-HAp and rhBMP-2/fgHAp ceramics

For the fg-HAp ceramics blocks, at 4 weeks after the implantation, body fluid extensively permeated into the bulk regions of HAp through the nano-micro-pores of the ceramics. Surface- and bulkdegradations of the HAp proceeded, so that a total size of the HAp block remarkably decreased. The volumetric populations occupied by body fluid were $60 \%$ at 4 weeks and $68 \%$ at 8 weeks in the ceramics explants, indicating drastic bioabsorption. At 12 weeks, the fg-HAp ceramics were completely biodegraded and bioabsorbed. The bioabsorption rate of the fg-HAp ceramics was much faster than that of the commercial porous $ß$-TCP ceramics. ${ }^{15)}$ No bone or cartilage was seen in any of the specimens. Investigating the eosinophilic region of HE sections marked in the fg-HAp ceramics, the immunohistochemical staining was carried out. The immunostaining of albumin in the fg-HAp explant tissue indicated to be immunopositive.

Figure 3 shows photomicrographs of histological sections at 4 weeks after the implantation of the rhBMP-2/fg-HAp ceramics. The HAp fragmentized by body fluid permeation and new bone formation from surface and bulk regions of the ceramics were observed. The HAp, which had been cut into small pieces, was incorporated into the induced bone with fatty marrow, suggesting that osteoinduction occurred with bone remodeling. The morphometric analysis conducted after the implantation indicated that the volumetric populations were $27 \%$ for HAp, 59\% for bone and marrow, and $14 \%$ for connective tissue.

\section{Discussion}

The calcination and partial dissolution-precipitation method proposed in this study is expected to produce a structure of fg$\mathrm{HAp}$, in which the composition ratios of r-HAp/b-HAp decrease from the surface layer of pore wall to a bulk region of the b-HAp body in the micropore matrix, forming gradations in both the crystallinity and the grain size of HAp. The fg-HAp ceramics with activated pore surfaces had a significantly higher specific surface area than the b-HAp ceramics. In the curves of the pore size distribution for the fg-HAp ceramics, pore volumes in the micro-pore sizes of 10-160 nm were surely recognized. The specified pore structure may be constructed in the macro-pores. Based on these results, it was found that the pore structure of the fg-HAp was classified into a macro-pore $(100-600 \mu \mathrm{m})$ originating from spongy bone and a micro-pore $(10-160 \mathrm{~nm})$ related to body fluid permeation and blood permeability. The structure of fg-HAp ceramics can permit body fluid to easily permeate the parts of a living body where it is used because of the number of graded micro-pores with nano-order. The easy permeation of body fluid into the fg-HAp structure implies better blood permeability, together with albumin included in blood. Microcracks in bulk regions of the ceramics formed by partial dissolution with $\mathrm{HNO}_{3}$ will be related to auto-degradation, body fluid permeation, and cellular invasion and proliferation. Consequently, the bioabsorbable and biomimetic fg-HAp ceramics may improve the blood permeability and bioabsorbability of bioceramics to advance the permeation rate of serum proteins in blood.

\section{Conclusions}

The absorbable fg-HAp ceramics with the specified pore structures and gradations in both crystallinity and grain size were newly developed by the calcination and partial dissolutionprecipitation methods. After implantation into the subcutaneous tissue of the back region of 4-week-old Wistar rats, the fg-HAp ceramics gave excellent bioabsorption characteristics because of the surface- and bulk- degradations of HAp grains, which were successively followed by body fluid permeation, blood permeability and albumin-adsorption. Concerning the rhBMP-2/ fg-HAp ceramics, permeation and diffusion of body fluid and active bone formation were observed at significant levels within 4 weeks of implantation. Accordingly, the rhBMP-2/fg-HAp ceramics can be a new biomimetic implant material exhibiting cellular absorption by giant cells or release characteristics of BMP, since the gradation conditions in crystallinity and grain size, accompanied by the needle-like pore wall of the fg-HAp would 
International symposium of Maxillofacial \& Oral Regenerative Biology in Okayama 2005

effectively control the bioabsorption rate and the permeation rate of body fluid.

\section{Reference}

1. Ogawa T., Chem. Chem. Indus. 58: Development of bone regeneration medicine system -Bone regeneration by hydroxyapatite ceramics-, 808-811, 2005

2. Niwa S., J. Japanese Soc. Biomaterials 23: Contribution to the development and clinical application of synthetic hydroxyapatite in the orthopedic field, 48-49, 2005

3. T. Ishimoto, T. Nakano, Y. Umakoshi, M. Yamamoto and Y. Tabata : Effects of applied stress on preferential alignment of biological apatite in rabbit forelimb bones, Phosphorus Res. Bull. 17: 77-82, 2004

4. Murata M., Akazawa T. and Arisue M.: Bone EngineeringBiological materials and bone morphogenetic proteins-, Phosphorus Res. Bull. 17: 51-58, 2004

5. Akazawa T., Murata M., Sasaki T., Tazaki J., Kobayashi M., Kanno T., Itabashi K., and Arisue M.: Preparation and characterization of bio-absorbable and functionally graded apatites originated from cattle bone, Proceedings of the 15th Symposium on FGM2003, 28-33, 2004

6. Murata M. and Akazawa T.: Fusion of cell biology and materials engineering-bone regeneration and biomaterials-, Phosphorus Lett. 47, 6-10, 2003

7. Okazaki M.: Apatites with trace elements, Phosphorus Res.

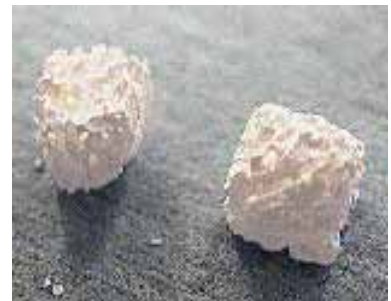

Fig. 1. Appearance of fg-HAp ceramics (3mm 3mm 3mm).
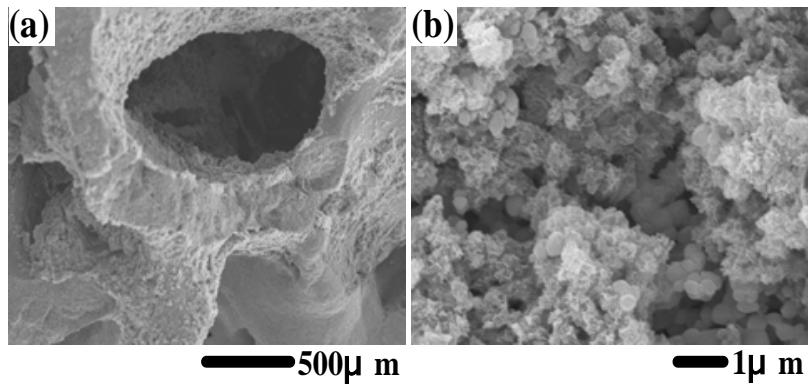

Fig. 2. SEM photographs of fg-HAp ceramics prepared by the calcination at $800 \bullet$ for $24 \mathrm{~h}$ and partial dissolution-precipitation. (a) Macro-pores with 100-600 $\mu \mathrm{m}$ were observed. (b) Spherical moss-like grains with 0.3$0.5 \mu \mathrm{m}$ forming needle-like microcrystals were seen in the surface and the macro-pores.
Bull. 17, 1-8, 2004

8. Joschek S., Nies B., Krotz R., Gopferich A.: Chemical and physicochemical characterization of porous hydroxyapatite ceramics made of natural bone, Biomaterials 21, 1645-1658, 2000

9. Akazawa T.: Production technology of cattle bone-originated apatites, J. High Temp. Soc. 104, 103-110, 2004

10. Murata M., Inoue M., Arisue M., Kuboki Y., and Nagai N.: Carrier-dependency of cellular differentiation induced by bone morphogenetic protein in ectopic sites, Int. J. Oral Maxillofac Surg 27, 391-396, 1998

11. Gao T., Lindholm TS., Marttinen A., and Urist MR. : Composites of bone morpho-genetic protein (BMP) and type IV collagen, coral - derived coral hydroxyapatite, and tricalcium phosphate ceramics, Int Orthop. 20, 5, 321-325, 1996

12. Nakashima M. and Reddi A.H.: The application of bone morphogenetic proteins to dental tissue engineering, Nature Biotech. 21, 9, 1025-1032, 2003

13. Oda S., Kinoshita A., Higuchi T., Ishikawa I: Ectopic bone formation by rhBMP-2 with various calcium phosphate ceramics, Dent. Res. 74, 574, 1995

14. Takahashi T., Tominaga T., Watabe N., Yokobori T., Sasada H., Yoshimoto T.: Use of porous hydroxyapatite graft containing recombinant human bone morphogenetic protein2 for cervical fusion in a caprine model, J. Neurosurg. 90, 224-230, 1999

15. Artzi Z, Weinreb M, Givol N, Rohrer MD, Nemcovsky CE, Prasad HS, Tal H, Biomaterial resorption rate and healing site morphology of inorganic bovine bone and beta-tricalcium phosphate in the canine: a 24-month longitudinal histologic study and morphometric analysis, Int. J Oral Maxillofac Implants 19, 3, 357-368, 2004

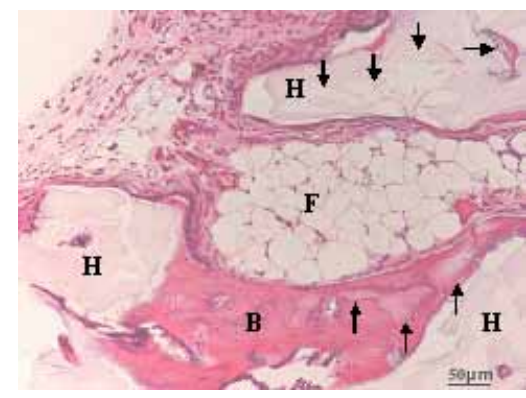

Fig. 3. Photomicrographs of HE sections at 4 weeks after the implantation of rh BMP-2/fg-HAp ceramics. B: bone, H: HAp containing body fluid, F: fatty marrow, $\downarrow$ : fragmentized HAp, $\uparrow$ : osteoinduction with bone remodeling. HAp fragmentized by body fluid permeation and new bone formation from surface and bulk regions of the ceramics were observed, while HAp incorporated by the induced bone with fatty marrow were found. 